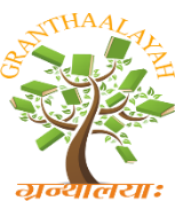

\author{
INTERNATIONAL JOURNAL OF RESEARCH \\ GRANTHAALAYAH \\ A knowledge Repository
}

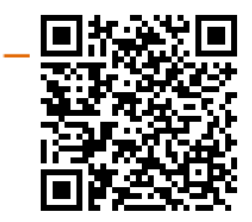

Science

\title{
REDUCTION OF ACTIVE POWER LOSS BY VOLITION PARTICLE SWARM OPTIMIZATION
}

\author{
Dr.K.Lenin *1 \\ ${ }^{* 1}$ Professor Prasad V. Potluri Siddhartha Institute of Technology Kanuru, Vijayawada, Andhra \\ Pradesh -520007, India
}

\begin{abstract}
This paper projects Volition Particle Swarm Optimization (VP) algorithm for solving optimal reactive power problem. Particle Swarm Optimization algorithm (PSO) has been hybridized with the Fish School Search (FSS) algorithm to improve the capability of the algorithm. FSS presents an operator, called as collective volition operator, which is capable to auto-regulate the exploration-exploitation trade-off during the algorithm execution. Since the PSO algorithm converges faster than FSS but cannot auto-adapt the granularity of the search, we believe the FSS volition operator can be applied to the PSO in order to mitigate this PSO weakness and improve the performance of the PSO for dynamic optimization problems. In order to evaluate the efficiency of the proposed Volition Particle Swarm Optimization (VP) algorithm, it has been tested in standard IEEE 30 bus test system and compared to other reported standard algorithms. Simulation results show that Volition Particle Swarm Optimization (VP) algorithm is more efficient then other algorithms in reducing the real power losses with control variables are within the limits..
\end{abstract}

Keywords: Optimal Reactive Power; Transmission Loss; Particle Swarm Optimization; Fish School Search Algorithm.

Cite This Article: Dr.K. Lenin (2018). "REDUCTION OF ACTIVE POWER LOSS BY VOLITION PARTICLE SWARM OPTIMIZATION.” International Journal of Research Granthaalayah, 6(6), 346-356. https://doi.org/10.29121/granthaalayah.v6.i6.2018.1379.

\section{Introduction}

Optimal reactive power problem is a multi-objective optimization problem that minimizes the real power loss and bus voltage deviation. Various mathematical techniques like the gradient method [1-2], Newton method [3] and linear programming [4-7] have been adopted to solve the optimal reactive power dispatch problem. Both the gradient and Newton methods have the complexity in managing inequality constraints. If linear programming is applied then the input- output function has to be uttered as a set of linear functions which mostly lead to loss of accuracy. The problem of voltage stability and collapse play a major role in power system planning and operation [8]. Global optimization has received extensive research awareness, and a great number of methods have been applied to solve this problem. 
Evolutionary algorithms such as genetic algorithm have been already proposed to solve the reactive power flow problem [9, 10]. Evolutionary algorithm is a heuristic approach used for minimization problems by utilizing nonlinear and non-differentiable continuous space functions. In [11], Genetic algorithm has been used to solve optimal reactive power flow problem. In [12], Hybrid differential evolution algorithm is proposed to improve the voltage stability index. In [13] Biogeography Based algorithm is projected to solve the reactive power dispatch problem. In [14], a fuzzy based method is used to solve the optimal reactive power scheduling method. In [15], an improved evolutionary programming is used to solve the optimal reactive power dispatch problem. In [16], the optimal reactive power flow problem is solved by integrating a genetic algorithm with a nonlinear interior point method. In [17], a pattern algorithm is used to solve ac-dc optimal reactive power flow model with the generator capability limits. In [18], F. Capitanescu proposes a two-step approach to evaluate Reactive power reserves with respect to operating constraints and voltage stability.

In [19], a programming based approach is used to solve the optimal reactive power dispatch problem. In [20], A. Kargarian et al present a probabilistic algorithm for optimal reactive power provision in hybrid electricity markets with uncertain loads. In general, swarm algorithms are inspired in groups of animals, such as flocks of birds, schools of fish, hives of bees, colonies of ants, etc. Although a lot of swarm-based algorithms were already proposed, just some few were designed to tackle dynamic problems. One of the most used swarm intelligence algorithms is the Particle Swarm Optimization (PSO). Despite the fast convergence capability, the vanilla version of the PSO cannot tackle dynamic optimization problems.

It occurs because the entire swarm often increases the exploitation around a good region of the search space, reducing the overall diversity of the population. However, some variations of the PSO have been created in order to increase the capacity to escape from regions in the search space where the optimum is not located anymore [21-25]. On the other hand, another swarm intelligence algorithm proposed in 2008, the Fish School Search algorithm (FSS) [26-28], presents a very interesting feature that can be very useful for dynamic environments. FSS presents an operator, called collective volition operator, which is capable to auto-regulate the exploration-exploitation trade-off during the algorithm execution. Since the PSO algorithm converges faster than FSS but cannot auto-adapt the granularity of the search, \& the FSS collective volition operator can be applied to the PSO in order to mitigate this PSO weakness and improve the performance of the PSO for dynamic optimization problems. In order to evaluate the efficiency of the proposed Volition Particle Swarm Optimization (VP) algorithm, it has been tested in standard IEEE 30 bus test system and compared to other reported standard algorithms. Simulation results show that Volition Particle Swarm Optimization (VP) algorithm is more efficient then other algorithms in reducing the real power losses with control variables are within the limits.

\section{Problem Formulation}

The optimal power flow problem is treated as a general minimization problem with constraints, and can be mathematically written in the following form:

Minimize $\mathrm{f}(\mathrm{x}, \mathrm{u})$ 
subject to $\mathrm{g}(\mathrm{x}, \mathrm{u})=0$

and

$h(x, u) \leq 0$

where $f(x, u)$ is the objective function. $g(x . u)$ and $h(x, u)$ are respectively the set of equality and inequality constraints. $\mathrm{x}$ is the vector of state variables, and $\mathrm{u}$ is the vector of control variables.

The state variables are the load buses (PQ buses) voltages, angles, the generator reactive powers and the slack active generator power:

$\mathrm{x}=\left(\mathrm{P}_{\mathrm{g} 1}, \theta_{2}, \ldots, \theta_{\mathrm{N}}, \mathrm{V}_{\mathrm{L} 1}, ., \mathrm{V}_{\mathrm{LNL}}, \mathrm{Q}_{\mathrm{g} 1}, \ldots, \mathrm{Q}_{\mathrm{gng}}\right)^{\mathrm{T}}$

The control variables are the generator bus voltages, the shunt capacitors/reactors and the transformers tap-settings:

$\mathrm{u}=\left(\mathrm{V}_{\mathrm{g}}, \mathrm{T}, \mathrm{Q}_{\mathrm{c}}\right)^{\mathrm{T}}$

or

$\mathrm{u}=\left(\mathrm{V}_{\mathrm{g} 1}, \ldots, \mathrm{V}_{\mathrm{gng}}, \mathrm{T}_{1}, \ldots, \mathrm{T}_{\mathrm{Nt}}, \mathrm{Q}_{\mathrm{c} 1}, \ldots, \mathrm{Q}_{\mathrm{cNc}}\right)^{\mathrm{T}}$

Where ng, nt and nc are the number of generators, number of tap transformers and the number of shunt compensators respectively.

\section{Objective Function}

\section{Active Power Loss}

The objective of the reactive power dispatch is to minimize the active power loss in the transmission network, which can be described as follows:

$F=P L=\sum_{k \in N b r} g_{k}\left(V_{i}^{2}+V_{j}^{2}-2 V_{i} V_{j} \cos \theta_{i j}\right)$

Or

$F=P L=\sum_{i \in N g} P_{g i}-P_{d}=P_{\text {gslack }}+\sum_{i \neq s l a c k}^{N g} P_{g i}-P_{d}$

Where gk: is the conductance of branch between nodes $\mathrm{i}$ and $\mathrm{j}$, Nbr: is the total number of transmission lines in power systems. Pd: is the total active power demand, Pgi: is the generator active power of unit $i$, and Pgsalck: is the generator active power of slack bus.

\section{Voltage Profile Improvement}

For minimizing the voltage deviation in PQ buses, the objective function becomes:

$F=P L+\omega_{v} \times V D$ 
Where $\omega \mathrm{v}$ : is a weighting factor of voltage deviation.

VD is the voltage deviation given by:

$V D=\sum_{i=1}^{N p q}\left|V_{i}-1\right|$

\section{Equality Constraint}

The equality constraint $\mathrm{g}(\mathrm{x}, \mathrm{u})$ of the Optimal reactive power problem is represented by the power balance equation, where the total power generation must cover the total power demand and the power losses:

$P_{G}=P_{D}+P_{L}$

This equation is solved by running Newton Raphson load flow method, by calculating the active power of slack bus to determine active power loss.

\section{Inequality Constraints}

The inequality constraints $\mathrm{h}(\mathrm{x}, \mathrm{u})$ reflect the limits on components in the power system as well as the limits created to ensure system security. Upper and lower bounds on the active power of slack bus, and reactive power of generators:

$P_{\text {gslack }}^{\min } \leq P_{\text {gslack }} \leq P_{\text {gslack }}^{\max }$

$Q_{g i}^{\min } \leq Q_{g i} \leq Q_{g i}^{\max }, i \in N_{g}$

Upper and lower bounds on the bus voltage magnitudes:

$V_{i}^{\min } \leq V_{i} \leq V_{i}^{\max }, i \in N$

Upper and lower bounds on the transformers tap ratios:

$T_{i}^{\min } \leq T_{i} \leq T_{i}^{\max }, i \in N_{T}$

Upper and lower bounds on the compensators reactive powers:

$Q_{c}^{\min } \leq Q_{c} \leq Q_{C}^{\max }, i \in N_{C}$

Where $\mathrm{N}$ is the total number of buses, NT is the total number of Transformers; Ncis the total number of shunt reactive compensators.

\section{Particle Swarm Optimization (Pso)}

Particle Swarm Optimization (PSO) is a population-based optimization algorithm inspired by the behaviour of flocks of birds. The standard approach is composed by a swarm of particles, where each one has a position within the search space $\overrightarrow{x_{\imath}}$ and each position represents a solution for the problem. The particles fly through the search space of the problem searching for the best solution, 
according to the current velocity $\vec{v}_{l}$ the best position found by the particle itself $\left(\overrightarrow{P_{\text {best }}}\right)$ and the best position found by the entire swarm during the search so far $\left(\overrightarrow{G_{\text {best }}}\right)$. According this approach is also called inertia PSO \& the velocity of a particle i is evaluated at iteration's of the algorithm by using the following equation,

$\left(\overrightarrow{v_{l}}(t+1)=\omega \overrightarrow{v_{l}}(t)+r_{1} c_{1}\left|\overrightarrow{P_{\text {best }}}-\overrightarrow{x_{l}}(t)\right|+r_{2} c_{2}\left|\overrightarrow{G_{\text {best }_{l}}}-\overrightarrow{x_{l}}(t)\right|\right.$

Where $r \_1$ and $r \_2$ are numbers randomly generated in the interval $[0,1]$. The inertia weight $(\omega)$ controls the influence of the previous velocity and balances the exploration-exploitation behaviour along the process. It generally decreases from 0.9 to 0.4 during the algorithm execution. c_1 \& c_2 are called cognitive and social acceleration constants, respectively, and weights the influence of the memory of the particle and the information acquired from the neighbourhood. The position of each particle is updated based on the velocity of the particle, according to the following equation:

$\overrightarrow{x_{l}}(t+1)=\overrightarrow{x_{l}}(t)+\overrightarrow{v_{l}}(t+1)$

The communication topology defines the neighbourhood of the particles and, as a consequence, the flow of information through the particles. There are two basic topologies: global and local. In the former, each particle shares and acquires information directly from all other particles, i.e. all particles use the same social memory, called Gbest. In the local topology, each particle only shares information with two neighbours and the social memory is not the same within the whole swarm. This approach, called Lbest, helps to avoid a premature attraction of all particles to a single spot point in the search space.

Since the standard PSO cannot tackle dynamic problems due to the low capacity to increase the diversity after the entire swarm has converge to a single region of the search space, many efforts to overcome this weakness have been made. The simplest idea is to restart the particles every time the search space changes. However, all the previous information obtained from the problem during the search process is lost in this case. An interesting approach is utilizing the idea of electrostatic charges. Some particles are charged (they repeal themselves) and some others are neutral. In general, the neutral particles tend to exploit towards a single sub-region of the search space, whereas the charged particles never converges to a unique spot. Nevertheless, the charged particles are constantly exploring in order to maintain diversity. In order to consider the effect of the charged particles, the velocity equation receives a fourth term, as shown in the equation (26). This term is defined as the acceleration of the particle $i\left(\overrightarrow{a_{l}}\right)$ and can be seen in equation (20).

$\overrightarrow{v_{l}}(t+1)=\omega \overrightarrow{v_{l}}(t)+r_{1} c_{1}\left|\overrightarrow{P_{\text {best }_{l}}}-\overrightarrow{x_{l}}(t)\right|+r_{2} c_{2}\left|\overrightarrow{G_{\text {best }_{l}}}-\overrightarrow{x_{l}}(t)\right|+\overrightarrow{a_{l}}(t)$

$\left(\overrightarrow{a_{l}}(t)=\left\{\begin{array}{l}\sum_{i \neq j} \frac{Q_{i} Q_{j}}{\left.\overrightarrow{\vec{r}_{l}}(t)\right|^{3}} \overrightarrow{r_{l j}}(t), \text { if } R_{e} \leq\left\|\overrightarrow{r_{l j}}(t)\right\| \leq R_{p} \\ 0, \text { otherwise }\end{array}\right.\right.$

Where $\overrightarrow{r_{l j}}(t)=\overrightarrow{x_{l}}(t)-\overrightarrow{x_{j}}(t), Q_{i}$ is the charge magnitude of the particle $\mathrm{i}, \mathrm{Re}$ is the core radius and $\mathrm{Rp}$ is the perception limit of the particle . Neutral particles have charge value equal to zero, i.e. $\mathrm{Qi}=0$. 


\section{Fish School Search (FSS)}

The Fish School Search (FSS) is an optimization algorithm based on the gregarious behaviour of oceanic fish. In the FSS, each fish represents a solution for the problem. The success of a fish during the search process is indicated by its weight. The FSS has four operators, which are executed for each fish of the school at each iteration: (i) individual movement, which is responsible for local search stepind; (ii) feeding, which updates the fish weights indicating the degree of success or failure during the search process so far; (iii) collective-instinctive movement, which makes all fish moves toward a resultant direction; and (iv) collective volition operator, which controls the granularity of the search. In this paper, as we are dealing with dynamic environments, only the feeding and collective volition operator movement operators are used to build the proposed algorithm.

\section{Feeding Operator}

The feeding operator determines the variation of the fish weight at iteration's. One should notice that a fish can increase or decrease its weight depending, respectively, on the success or failure during the search process. The weight of the fish is evaluated according to the following equation:

$W_{i}(t+1)=W_{i}(t)+\frac{\Delta f_{i}}{\max (|\Delta f|)}$,

Where $W_{i}(t)$ is the weight of the fish $\Delta f_{i}$ is the variation of the fitness function between the new position and the current position of the fish, $\max (|\Delta \mathrm{f}|)$ is the absolute value of the greatest fitness variation among all fish. There is a parameter wscale that limits the maximum weight of the fish. The weight of each fish can vary between 1 and wscale and has an initial value equal to $\frac{W_{\text {scale }}}{2}$

\section{Collective-Volitive Movement Operator}

This operator controls the granularity of the search executed by the fish school. When the whole school is achieving better results, the operator approximates the fish aiming to accelerate the convergence toward a good region. On the contrary, the operator spreads the fish away from the barycenter of the school and the fish have more chances to escape from a local minimum. The fish school expansion or contraction is applied as a small drift to every fish position regarding the school barycenter, which can be evaluated as shown below:

$\vec{B}(t)=\frac{\sum_{i=1}^{N} \overrightarrow{x_{l}}(t) W_{i}(t)}{\sum_{i=1}^{N} \overrightarrow{x_{l}}(t)}$

We use equation (23) to perform the fish school expansion (use sign + ) or contraction (use sign $-)$.

$\overrightarrow{x_{l}}(t+1)=\overrightarrow{x_{l}}(t) \pm \operatorname{step}_{v o l}{ }^{r 1} \frac{\overrightarrow{x_{l}}(t)-\vec{B}(t)}{d\left(\overrightarrow{x_{l}}(t), \vec{B}(t)\right)}$

Where $\mathrm{r} 1$ is a number randomly generated in the interval $[0,1] . d\left(\overrightarrow{x_{l}}, \vec{B}\right)$ Evaluates the euclidean distance between the particles $i$ and the barycenter. stepvol is called volition step and controls the step size of the fish. The stepvol is bounded by two parameters (stepvol_min and stepvol_max) and decreases linearly from stepvol max to stepvol_min along the algorithm iterations. It helps the 
algorithm to initialize with exploration behaviour and change dynamically to exploitation behaviour.

\section{Volition Particle Swarm Optimization (VP) For Solving Optimal Reactive Power Problem}

Particle Swarm Optimization algorithm (PSO) has been hybridized with the Fish School Search (FSS) algorithm to improve the capability of the algorithm. Proposed approach includes two FSS operators in the Inertia PSO, the feeding and the collective-Volition movement. In the PSO, each particle becomes a weighted particle, where the weight is used to indicate the collective- Volition movement, resulting in expansion or contraction of the school. In our proposal, the stepvol does not decrease linearly; it decreases according to equation (24). The parameter Volition step decay percentage (decayvol) must be in the interval $[0,100]$.

step $_{v o l}(t+1)=\operatorname{step}_{v o l}(t) \frac{100-\text { decay }_{v o l}}{100}$

The stepvol is reinitialized to stepvol_max when a change in the environment is detected. The fitness of the sentry particle is evaluated in the end iteration's and in the beginning of the next iteration.

Initialize parameters and particles;

While the stop condition is not reached do

For each particle of the swarm do

Evaluate the fitness of the particle;

Evaluate $\left(\overrightarrow{P_{\text {best }}}\right)$ and $\left(\overrightarrow{L_{\text {best }}}\right)$

End

if an environment change is detected then

Initialize stepvol;

End

For each particle of the swarm do

Update the velocity and the position of the particle;

Evaluate the fitness of the particle;

End

Execute feeding operator;

Execute collective-Volition movement operator;

For each particle of the swarm do

Evaluate $\left(\overrightarrow{P_{\text {best }}}\right)$ and $\left(\overrightarrow{L_{\text {bestl }}}\right)$;

End

Update stepvol and w;

End

\section{Simulation Results}

Validity of the proposed Volition Particle Swarm Optimization (VP) algorithm has been verified by testing in IEEE 30-bus, 41 branch system and it has 6 generator-bus voltage magnitudes, 4 transformer-tap settings, and 2 bus shunt reactive compensators. Bus 1 is taken as slack bus and 2, 
5, 8, 11 and 13 are considered as PV generator buses and others are PQ load buses. Control variables limits are given in Table 1.

Table 1: Primary Variable Limits $(\mathrm{Pu})$

\begin{tabular}{|l|l|l|l|}
\hline Variables & Min. & Max. & category \\
\hline Generator Bus & 0.95 & 1.1 & Continuous \\
\hline Load Bus & 0.95 & 1.05 & Continuous \\
\hline Transformer-Tap & 0.9 & 1.1 & Discrete \\
\hline Shunt Reactive Compensator & -0.11 & 0.31 & Discrete \\
\hline
\end{tabular}

In Table 2 the power limits of generators buses are listed.

Table 2: Generators Power Limits

\begin{tabular}{|l|l|l|l|l|l|}
\hline Bus & Pg & Pgmin & Pgmax & Qgmin & Qmax \\
\hline 1 & 96.00 & 49 & 200 & 0 & 10 \\
\hline 2 & 79.00 & 18 & 79 & -40 & 50 \\
\hline 5 & 49.00 & 14 & 49 & -40 & 40 \\
\hline 8 & 21.00 & 11 & 31 & -10 & 40 \\
\hline 11 & 21.00 & 11 & 28 & -6 & 24 \\
\hline 13 & 21.00 & 11 & 39 & -6 & 24 \\
\hline
\end{tabular}

Table 3 shows the proposed Volition Particle Swarm Optimization (VP) algorithm successfully kept the control variables within limits. Table 4 narrates about the performance of the proposed Volition Particle Swarm Optimization (VP) algorithm. Table 5 list out the overall comparison of the results of optimal solution obtained by various methods.

Table 3: After optimization values of control variables

\begin{tabular}{|l|l|}
\hline Control Variables & VP \\
\hline V1 & 1.0386 \\
\hline V2 & 1.0392 \\
\hline V5 & 1.0184 \\
\hline V8 & 1.0226 \\
\hline V11 & 1.0684 \\
\hline V13 & 1.0382 \\
\hline T4,12 & 0.00 \\
\hline T6,9 & 0.01 \\
\hline T6,10 & 0.90 \\
\hline T28,27 & 0.91 \\
\hline Q10 & 0.10 \\
\hline Q24 & 0.10 \\
\hline Real power loss & 4.2542 \\
\hline Voltage deviation & 0.9086 \\
\hline
\end{tabular}


Table 4: Performance of VP algorithm

\begin{tabular}{|l|l|}
\hline Iterations & 29 \\
\hline Time taken (secs) & 8.42 \\
\hline Real power loss & 4.2542 \\
\hline
\end{tabular}

Table 5: Comparison of results

\begin{tabular}{|l|l|}
\hline Techniques & Real power loss (MW) \\
\hline SGA(Wu et al., 1998) [29] & 4.98 \\
\hline PSO(Zhao et al., 2005) [30] & 4.9262 \\
\hline \multicolumn{1}{|c|}{ LP(Mahadevan et al., 2010) [31] } & 5.988 \\
\hline EP(Mahadevan et al., 2010) [31] & 4.963 \\
\hline CGA(Mahadevan et al., 2010) [31] & 4.980 \\
\hline AGA(Mahadevan et al., 2010) [31] & 4.926 \\
\hline CLPSO(Mahadevan et al., 2010) [31] & 4.7208 \\
\hline HSA (Khazali et al., 2011) [32] & 4.7624 \\
\hline BB-BC (Sakthivel et al., 2013) [33] & 4.690 \\
\hline MCS(Tejaswini sharma et al.,2016) [34] & 4.87231 \\
\hline Proposed VP & 4.2542 \\
\hline
\end{tabular}

\section{Conclusion}

In this paper a novel approach Volition Particle Swarm Optimization (VP) algorithm succesfully solved optimal reactive power problem. PSO algorithm converges faster than FSS but cannot autoadapt the granularity of the search, \& the FSS collective volition operator can be applied to the PSO in order to mitigate this PSO weakness and improve the performance of the PSO for dynamic optimization problems. In order to evaluate the efficiency of the proposed Volition Particle Swarm Optimization (VP) algorithm, it has been tested in standard IEEE 30 bus test system and compared to other reported standard algorithms. Simulation results show that Volition Particle Swarm Optimization (VP) algorithm is more efficient than other algorithms in reducing the real power losses with control variables are within the limits.

\section{References}

[1] O.Alsac,and B. Scott, "Optimal load flow with steady state security", IEEE Transaction. PAS 1973, pp. 745-751.

[2] Lee K Y, Paru Y M, Oritz J L -A united approach to optimal real and reactive power dispatch, IEEE Transactions on power Apparatus and systems 1985: PAS-104: 1147-1153

[3] A.Monticelli, M .V.F Pereira, and S. Granville, "Security constrained optimal power flow with post contingency corrective rescheduling", IEEE Transactions on Power Systems :PWRS-2, No. 1, pp.175-182.,1987.

[4] Deeb N, Shahidehpur S.M, Linear reactive power optimization in a large power network using the decomposition approach. IEEE Transactions on power system 1990: 5(2): 428-435

[5] E. Hobson,' Network consrained reactive power control using linear programming, 'IEEE Transactions on power systems PAS -99 (4), pp 868=877, 1980

[6] K.Y Lee, Y.M Park, and J.L Oritz, "Fuel -cost optimization for both real and reactive power dispatches", IEE Proc; 131C, (3), pp.85-93. 
[7] M.K. Mangoli, and K.Y. Lee, "Optimal real and reactive power control using linear programming", Electr.Power Syst.Res, Vol.26, pp.1-10,1993.

[8] C.A. Canizares, A.C.Z.de Souza and V.H. Quintana, "Comparison of performance indices for detection of proximity to voltage collapse,'” vol. 11. no.3, pp.1441-1450, Aug 1996.

[9] K.Anburaja, "Optimal power flow using refined genetic algorithm", Electr. Power Compon.Syst , Vol. 30, 1055-1063,2002.

[10] D. Devaraj, and B. Yeganarayana, "Genetic algorithm based optimal power flow for security enhancement", IEE proc-Generation. Transmission and. Distribution; 152, 6 November 2005.

[11] Berizzi, C. Bovo, M. Merlo, and M. Delfanti, "A ga approach to compare orpf objective functions including secondary voltage regulation," Electric Power Systems Research, vol. 84, no. 1, pp. 187 $-194,2012$.

[12] C.-F. Yang, G. G. Lai, C.-H. Lee, C.-T. Su, and G. W. Chang, "Optimal setting of reactive compensation devices with an improved voltage stability index for voltage stability enhancement," International Journal of Electrical Power and Energy Systems, vol. 37, no. 1, pp. 50 - 57, 2012.

[13] P. Roy, S. Ghoshal, and S. Thakur, "Optimal var control for improvements in voltage profiles and for real power loss minimization using biogeography-based optimization," International Journal of Electrical Power and Energy Systems, vol. 43, no. 1, pp. 830 - 838, 2012.

[14] B. Venkatesh, G. Sadasivam, and M. Khan, "A new optimal reactive power scheduling method for loss minimization and voltage stability margin maximization using successive multi-objective fuzzy lp technique," IEEE Transactions on Power Systems, vol. 15, no. 2, pp. 844 - 851, may 2000.

[15] W. Yan, S. Lu, and D. Yu, "A novel optimal reactive power dispatch method based on an improved hybrid evolutionary programming technique," IEEE Transactions on Power Systems, vol. 19, no. 2, pp. 913 - 918, may 2004.

[16] W. Yan, F. Liu, C. Chung, and K. Wong, "A hybrid genetic algorithm interior point method for optimal reactive power flow," IEEE Transactions on Power Systems, vol. 21, no. 3, pp. 1163 1169, aug. 2006.

[17] J. Yu, W. Yan, W. Li, C. Chung, and K. Wong, "An unfixed piecewise optimal reactive powerflow model and its algorithm for ac-dc systems," IEEE Transactions on Power Systems, vol. 23, no. 1 , pp. $170-176$, feb. 2008.

[18] F. Capitanescu, "Assessing reactive power reserves with respect to operating constraints and voltage stability," IEEE Transactions on Power Systems, vol. 26, no. 4, pp. 2224-2234, nov. 2011.

[19] Z. Hu, X. Wang, and G. Taylor, "Stochastic optimal reactive power dispatch: Formulation and solution method," International Journal of Electrical Power and Energy Systems, vol. 32, no. 6, pp. $615-621,2010$.

[20] Kargarian, M. Raoofat, and M. Mohammadi, "Probabilistic reactive power procurement in hybrid electricity markets with uncertain loads," Electric Power Systems Research, vol. 82, no. 1, pp. 68 $-80,2012$.

[21] Blackwell, T.M., Bentley, P.J.: "Dynamic Search with Charged Swarms. In: Proceedings of the Genetic and Evolutionary Computation", Conference, pp. 19-26 (2002)

[22] Rakitianskaia, A., Engelbrecht, A.P.: "Cooperative charged particle swarm optimiser. In: Congress on Evolutionary Computation”, CEC 2008, pp. 933-939 (June 2008)

[23] Nickabadi, A., Ebadzadeh, M.M., Safabakhsh, R.: "Evaluating the performance of DNPSO in dynamic environments", In: IEEE International Conference on Systems, Man and Cybernetics, pp. 2640-2645 (October 2008)

[24] Kennedy, J., Eberhart, R.C.: "Particle swarm optimization", In: Proceedings of IEEE international conference on neural networks, vol. 4, pp. 1942-1948 (1995).

[25] Shi, Y., Eberhart, R.: "A modified particle swarm optimizer.", In: The 1998 IEEE International Conference on Evolutionary Computation Proceedings, IEEE World Congress on Computational Intelligence, pp. 69-73 (1998). 
[26] Bastos-Filho, C.J.A., Neto, F.B.L., Lins, A.J.C.C., Nascimento, A.I.S., Lima, M.P.: “A novel search algorithm based on fish school behavior", In: IEEE International Conference on Systems, Man and Cybernetics, pp. 2646-2651. IEEE, Los Alamitos (October 2009)

[27] Bastos-Filho, C.J.A., Neto, F.B.L., Sousa, M.F.C., Pontes, M.R.: "On the Influence of the Swimming Operators in the Fish School Search Algorithm”, In: SMC, pp. 5012-5017 (October 2009)

[28] Bastos-Filho, C.J.A., de Lima Neto, F.B., Lins, A.J.C.C., Nascimento, A.I.S., Lima, M.P.: "Fish school search", In: Chiong, R. (ed.) Nature-Inspired Algorithms for Optimisation. SCI, vol. 193, pp. 261-277. Springer, Heidelberg (2009).

[29] Wu.Q.H,Y.J.Cao,andJ.Y.Wen,(1998), “Optimal reactive power dispatch using an adaptive genetic algorithm”, Int.J.Elect.Power Energy Syst. Vol 20. Pp. 563-569.

[30] Zhao.B,C.X.Guo,andY.J.CAO,(2005),“Multiagent-based particle swarm optimization approach for optimal reactive power dispatch”,IEEE Trans. Power Syst. Vol. 20, no. 2, pp. 1070-1078.

[31] Mahadevan.K,KannanP.S,(2010)“Comprehensive Learning Particle Swarm Optimization for Reactive Power Dispatch”, Applied Soft Computing, Vol. 10, No. 2, pp. 641-52.

[32] Khazali.A.H,M.Kalantar,(2011),“Optimal Reactive Power Dispatch based on Harmony Search Algorithm", Electrical Power and Energy Systems, Vol. 33, No. 3, pp. 684-692.

[33] Sakthivel.S,M.Gayathri,V.Manimozhi,(2013), “A Nature Inspired Optimization Algorithm for Reactive Power Control in a Power System", InternationalJournalofRecentTechnologyandEngineering,pp29-33Vol.2,Issue-1.

[34] Tejaswini Sharma,Laxmi Srivastava,Shishir Dixit (2016). "Modified Cuckoo Search Algorithm For Optimal Reactive Power Dispatch", Proceedings of 38 th IRF International Conference, pp48. 20th March, 2016, Chennai, India, ISBN: 978-93-85973-76-5

\footnotetext{
*Corresponding author.

E-mail address: gklenin@gmail.com
} 